\title{
LABVIEW-BASED FUZZY CONTROLLER DESIGN OF A LIGHTING CONTROL SYSTEM
}

Mou-Lin Jin

Department of Mechanical and Mechatronic Engineering, National Taiwan Ocean University, Keelung, Taiwan, R.O.C., b0100@mail.ntou.edu.tw

Ming-Chun Ho

Department of Mechanical and Mechatronic Engineering, National Taiwan Ocean University, Keelung, Taiwan, R.O.C.

Follow this and additional works at: https://jmstt.ntou.edu.tw/journal

Part of the Mechanical Engineering Commons

\section{Recommended Citation}

Jin, Mou-Lin and Ho, Ming-Chun (2009) "LABVIEW-BASED FUZZY CONTROLLER DESIGN OF A LIGHTING CONTROL SYSTEM," Journal of Marine Science and Technology. Vol. 17: Iss. 2, Article 5.

DOI: $10.51400 / 2709-6998.1965$

Available at: https://jmstt.ntou.edu.tw/journal/vol17/iss2/5

This Research Article is brought to you for free and open access by Journal of Marine Science and Technology. It has been accepted for inclusion in Journal of Marine Science and Technology by an authorized editor of Journal of Marine Science and Technology. 


\title{
LABVIEW-BASED FUZZY CONTROLLER DESIGN OF A LIGHTING CONTROL SYSTEM
}

\author{
Mou-Lin Jin* and Ming-Chun Ho
}

Key words: LabVIEW, lighting control system, MATLAB Fuzzy Logic Toolbox, fuzzy logic controller, SISO, DISO.

\begin{abstract}
This paper describes how we design a lighting control system including hardware and software. Hardware includes light sensing circuit, control circuit, and 8255 expanding I/O circuit, PC, and bulb. Sensing circuit uses photo-resistance component to sense the environmental light and then transmit the signal of the lightness to the computer through an 8-bit $\mathrm{A} / \mathrm{D}$ converter 0804 . The control circuit applies reed relay in digital control way to adjust the variable resistor value of the traditional dimmer. Software incorporates LABVIEW graphical programming language and MATLAB Fuzzy Logic Toolbox to design the light fuzzy controller. The rule-base of the fuzzy logic controller either for the single input single output (SISO) system or the double inputs single output (DISO) system is developed and compared based on the operation of the bulb and the light sensor. The control system can dim the bulb automatically according to the environmental light. It can be applied to many fields such as control of streetlights and lighting control of car's headlights and it is possible to save energy by dimming the bulb. Experimental results show that the fuzzy controller with the DISO system can make bulb response faster than with the SISO system under sudden change of environmental light.
\end{abstract}

\section{INTRODUCTION}

After Lotfi Zadeh had introduced the fuzzy logic in 1965, the fuzzy control method is extensively used since it has the advantage of being model-free without any a priori information required. It is easy to design a fuzzy control system with requisite knowledge and the experience of a skilled operator. Many issues focus on determining fuzzy control rules, membership functions, and structures of fuzzy controllers $[2,4,6]$. Various ways of fuzzy logic used to improve industrial control [5] and methodology to reduce the number of variables and the

Paper submitted 12/28/07; accepted 04/10/08. Author for correspondence: Mou-Lin Jin (e-mail: b0100@mail.ntou.edu.tw).

*Department of Mechanical and Mechatronic Engineering, National Taiwan Ocean University, Keelung, Taiwan, R.O.C.

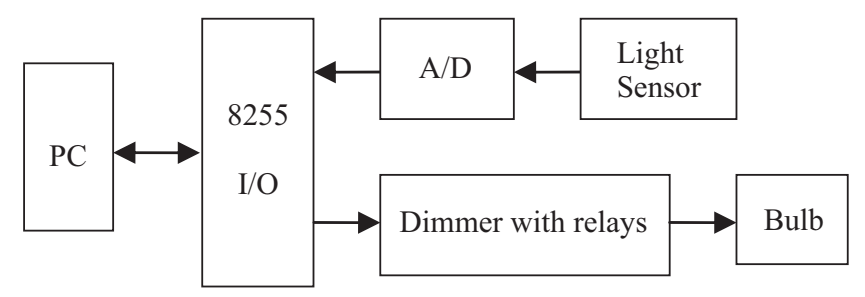

Fig. 1. The block diagram of the proposed lighting control system.

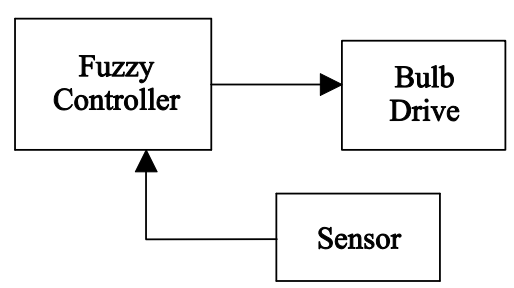

Fig. 2. The fuzzy control scheme.

number of fuzzy if-then rules [3] were discussed.

The application of fuzzy logic in lighting control has been presented in a number of papers. For example, it is possible to save energy by dimming the bulb or the fluorescent lamp. Dimming of the fluorescent lamp can be done by changing the input frequency of the electronic ballast [1]. A microprocessor-based intelligent control device for streetlight control applying fuzzy decision theory to distinguish various interferences accurately and to make it operate reliably, which can turn on or off the transformer automatically according to environmental light [7].

Section II introduces the proposed lighting control system including hardware design. One of the important problems involved with the design of fuzzy logic controllers is the development of fuzzy if-then rules for fuzzy controllers. Section III presents the design method of fuzzy logic controllers. The experimental results and discussions are shown in Section IV.

\section{SYSTEM DESCRIPTION}

The block diagram of the proposed lighting control system is shown in Fig. 1, which can be represented by a simple form like Fig. 2. The 8255 I/O shown in Fig. 1 is the expanding I/O circuit of a PC printer port. Originally, only 8-bit I/O in PC 

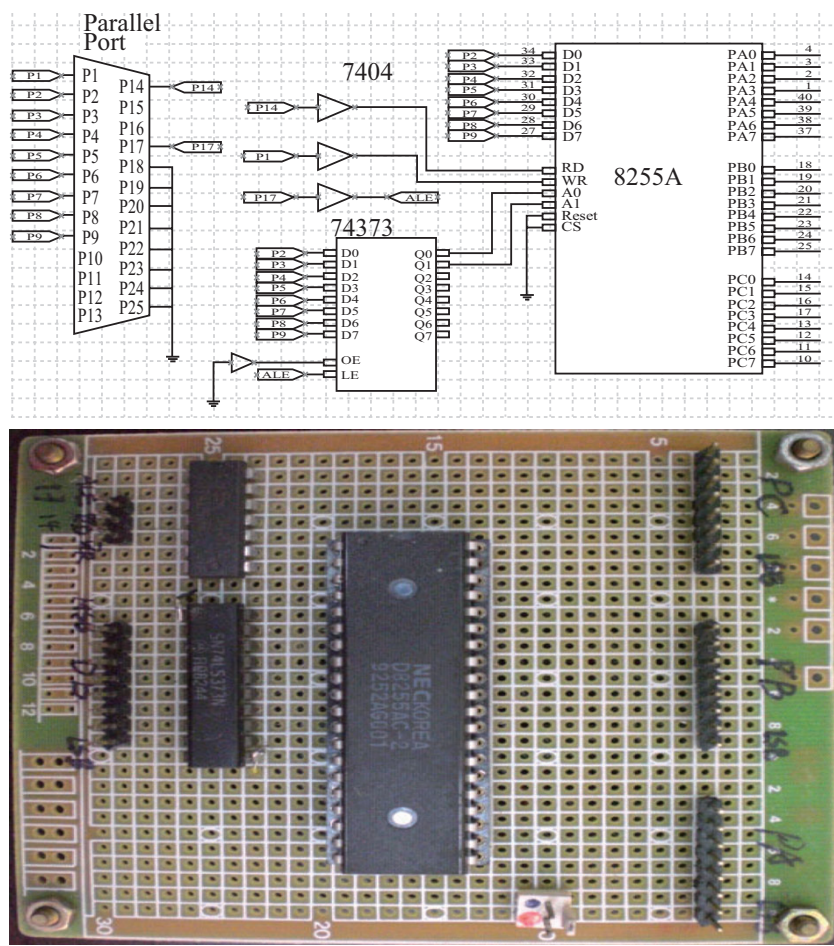

Fig. 3. The 8255 expanding I/O circuit connected with a PC printer port.

printer port can be used. After the 8255 expanding I/O circuit shown in Fig. 3 had been established, we have 24-bit I/O available now. The bulb drive shown in Fig. 2 is basically composed of a dimmer with 6 reed relays, and its corresponding hardware circuit is shown in Fig. 4. The reed relays in the bulb drive are regarded to as digital outputs of the light control system, thus the D/A converter is unnecessary for our case. One more thing need to be done is to design a parallel set of resistor circuit work as the function of the variable resistor in the dimmer. The main objective of designing a parallel-resistor circuit is to make the total resistance decreases homogeneously with the increase of the DN value of the control output. Figure 5 shows the hardware layout of the parallel-resistor circuit, and it stands for one of the many possible solutions as long as the above requirement (homogeneously decreasing) can be met. The simulation result of the total resistance versus control output is shown in Fig. 6. The sensor block in Fig. 2 corresponds to the combination of A/D block and the Light sensor block, its hardware circuit is shown in Fig. 7, which contains photo-resistor CdS sensor and an 8-bit A/D converter and LED displaying circuit. Consequently, the hardware layout of the lighting control system is shown in Fig. 8. After all the hardware works had been done, subsequently, our goal is to design a FLC used to dim the bulb according to the environmental light.

\section{FUZZY LOGIC CONTROLLER}

In this paper, the single input single output (SISO) system and
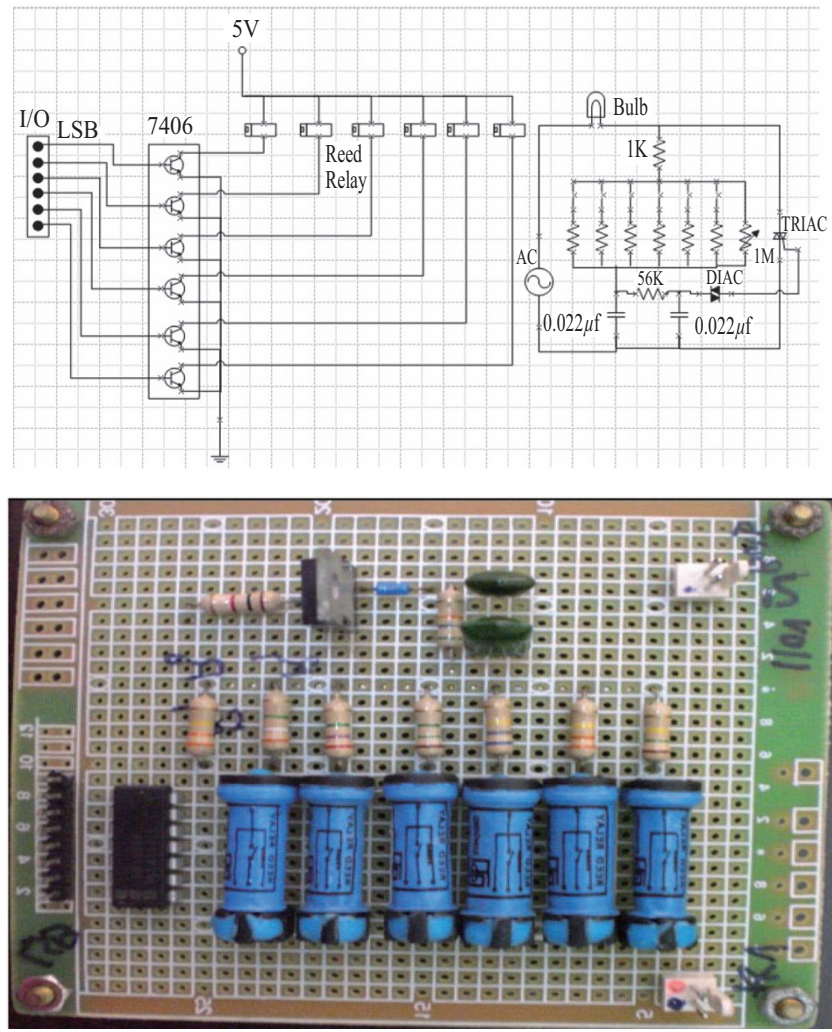

Fig. 4. The control circuit of the system.

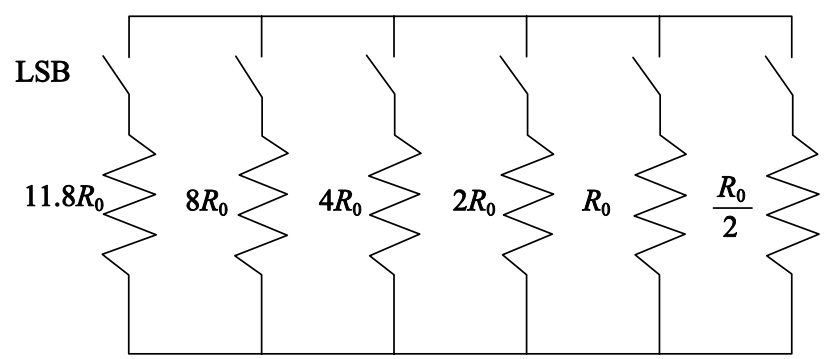

Fig. 5. The hardware layout of the parallel-resistor circuit $\left(R_{0}=330 K \Omega\right)$.

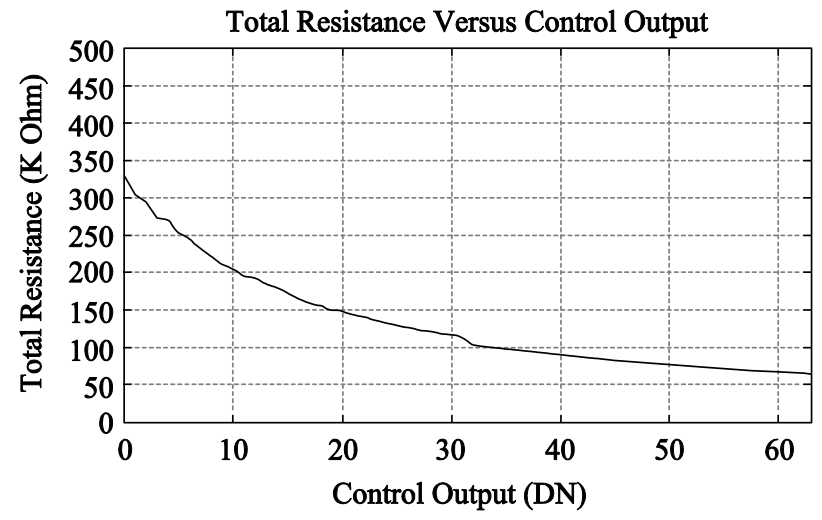

Fig. 6. Total resistance versus control output (DN: 0 63).

the double inputs single output (DISO) system are discussed. The environmental light is used as input variable $x_{1}$ for the SISO system, while another input variable $x_{2}$ (the changing 

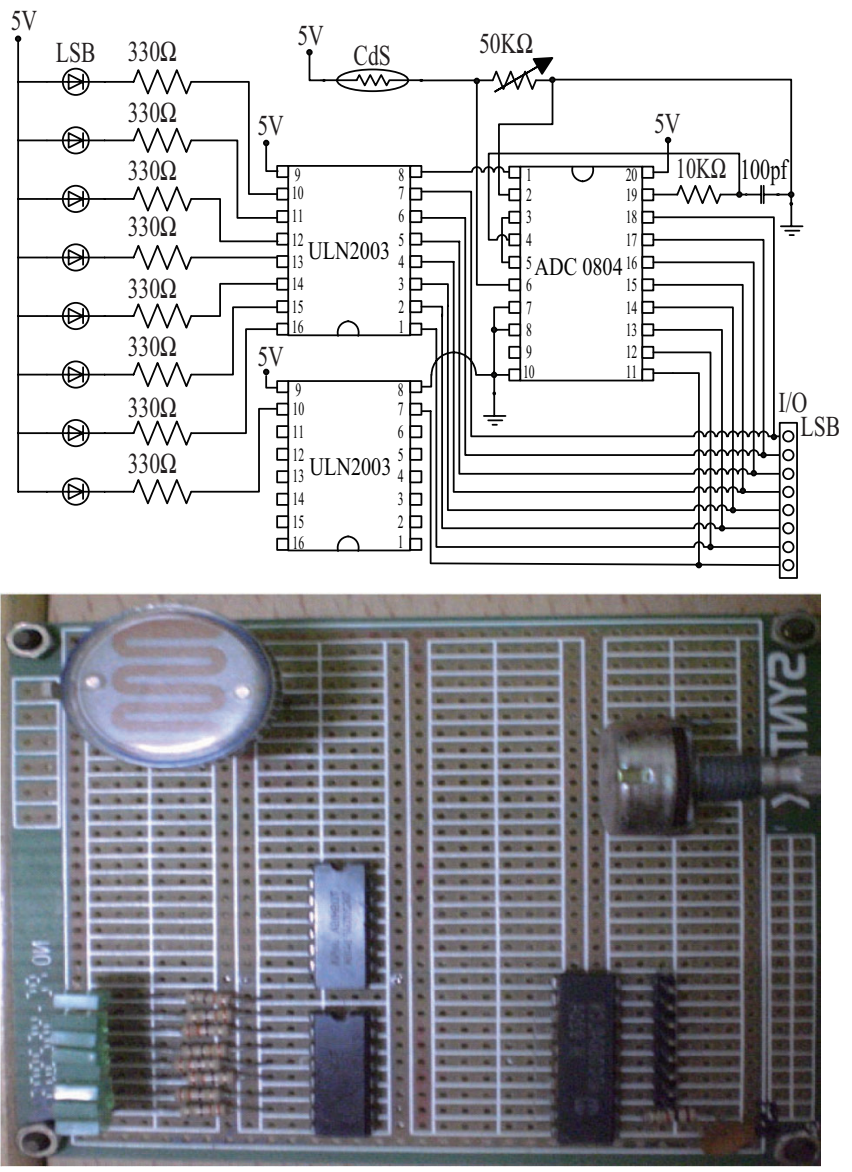

Fig. 7. The sensing circuit of the system.

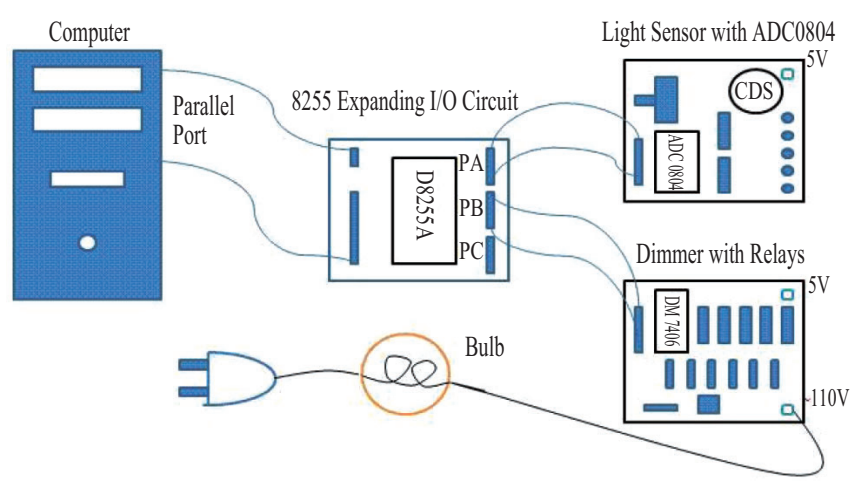

Fig. 8. The hardware layout of the lighting control system.

rate of the environmental light) is considered for the DISO system. The output variable is the numerical value DN to the output circuit. The input variables are fuzzified by assigning them a singleton fuzzy set, i.e. a set with membership function $\mu$ and zero elsewhere. The fuzzy set of the output variable is inferred by the max-min composition and the fuzzy relation describes the desired control action. The fuzzy set of the output variable is defuzzified to deliver a crisp numerical value by the center-of-gravity method. The fuzzy rule base consists of a collection of fuzzy IF-THEN rules of the form.
Table 1. Labels for the membership functions in the SISO system.

\begin{tabular}{cl}
\hline $\mathrm{S}$ & Small \\
$\mathrm{M}$ & Medium \\
$\mathrm{B}$ & Big \\
\hline
\end{tabular}

Table 2. Rule base of the SISO system.

\begin{tabular}{llll}
\hline$x_{1}$ & $\mathrm{~S}$ & $\mathrm{M}$ & $\mathrm{B}$ \\
\hline & $\mathrm{B}$ & $\mathrm{M}$ & $\mathrm{S}$ \\
\hline
\end{tabular}

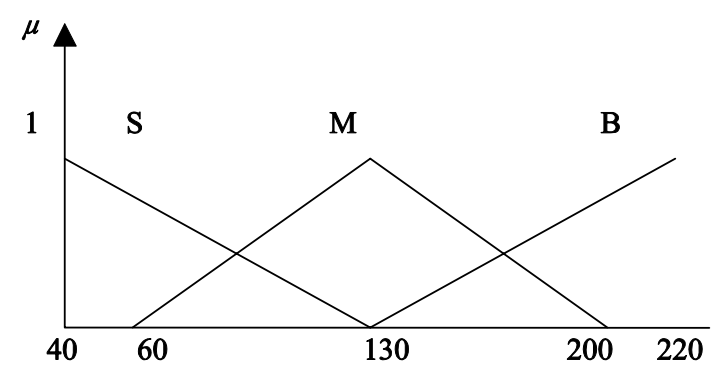

Environmental light $x_{1}$

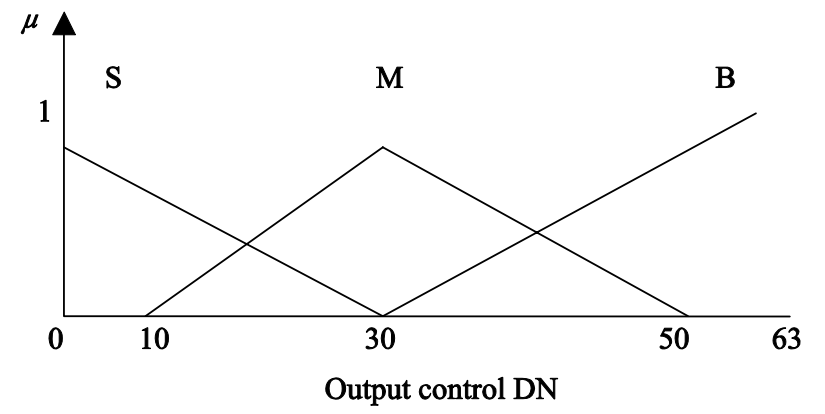

Fig. 9. Fuzzy sets showing the input and the output of the SISO system.

$$
\begin{aligned}
& R^{(k)}: \mathrm{IF} x_{1} \text { is and } x_{2} \text { is } F_{2}^{k}, \text { THEN y is } G^{k}, \\
& \text { for } \mathrm{k}=1,2, \ldots, \mathrm{n}
\end{aligned}
$$

Where $x_{1}, x_{2} \in U$, and $y \in R$ are the input and output of the fuzzy logic system, respectively, $F_{1}^{k}, F_{2}^{k}$ and $G^{k}$ are labels of fuzzy sets in $U_{1}, U_{2}$ and $R$ representing the $k^{\text {th }}$ antecedent pairs and consequent pair respectively and $\mathrm{n}$ is the number of rules.

The SISO system and the DISO system will be discussed and analyzed by using MATLAB Fuzzy Logic Toolbox in the followings.

\section{SISO}

We assume that the input to the system is the environmental light $x_{1}$. We further assume that the environmental light can be Big, Medium, and Small. The output DN can range between 0 and 63 and is divided into Small, Medium, and Big. Figure 9 shows the fuzzy sets describing the above. Labels for the membership functions are given in Table 1. The rules base, with its 3 rules, is shown in Table 2. 


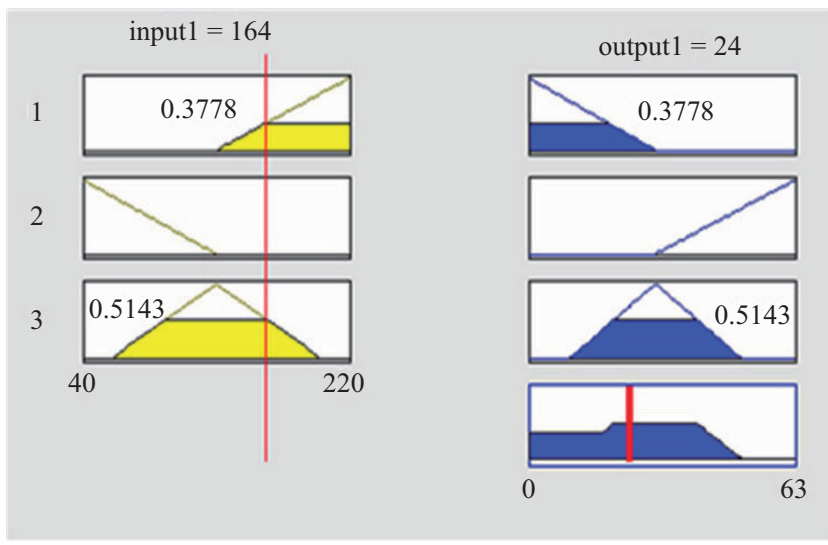

Fig. 10. The inferred output of the SISO system.
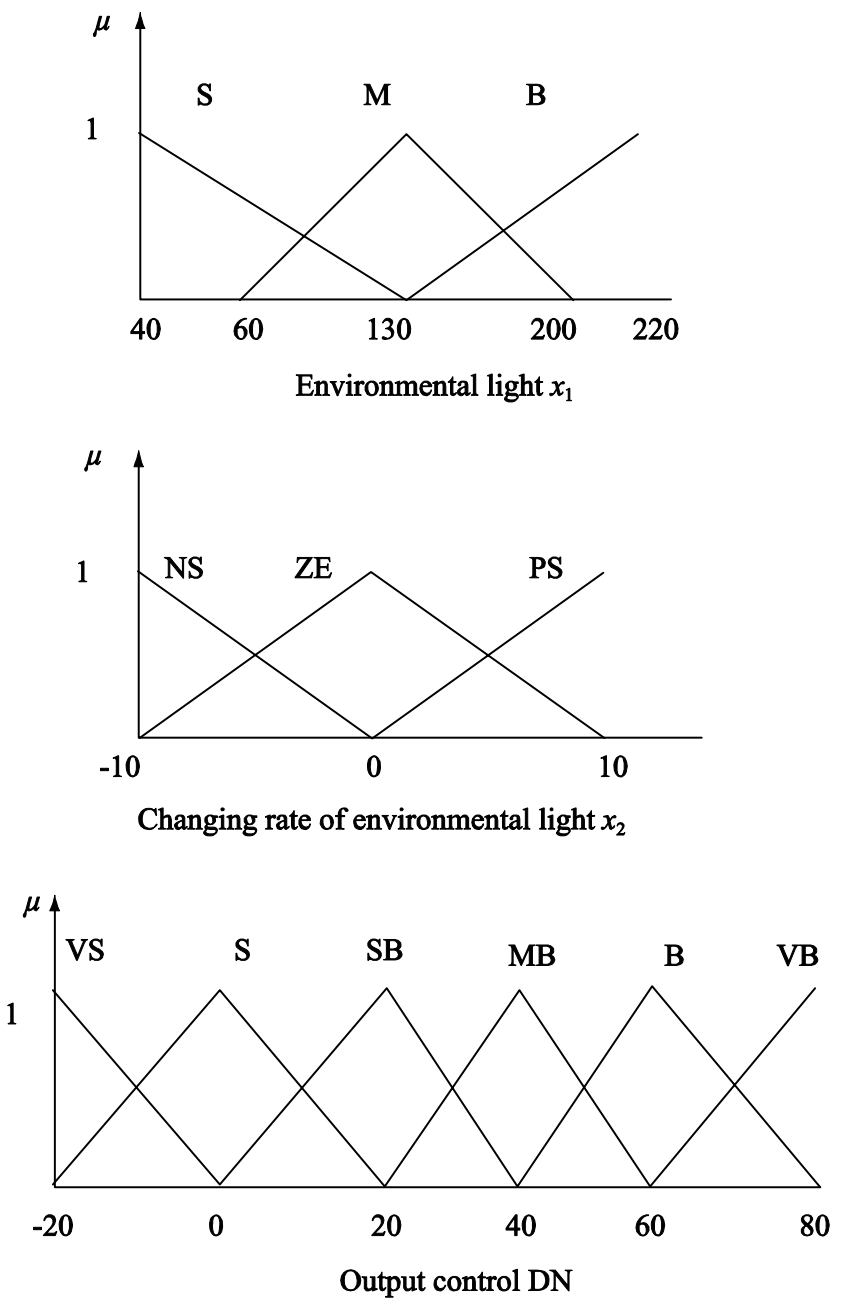

Fig. 11. Fuzzy sets showing the inputs and the output of the DISO system.

Figure 10 shows the results of the simulation of the rule base by fuzzy inference development environment (MATLAB Fuzzy Logic Toolbox) software for the input value of the environmental light $x_{1}=164$. The output is 24.22 . Here only two rules are needed to calculate the output. The inferred
Table 3. Labels for the membership functions in the DISO system.

\begin{tabular}{llll}
\hline VS & Very Small & NS & Negative Small \\
\hline S & Small & ZE & Zero \\
SB & Small Big & PS & Positive Small \\
MB & Medium Big & & \\
\hline B & Big & & \\
\hline VB & Very Big & & \\
\hline
\end{tabular}

Table 4. Rule base of the DISO system.

\begin{tabular}{llll}
\hline$x_{1} / x_{2}$ & $\mathrm{~S}$ & $\mathrm{M}$ & $\mathrm{B}$ \\
\hline $\mathrm{PS}$ & $\mathrm{MB}$ & $\mathrm{S}$ & $\mathrm{VS}$ \\
$\mathrm{ZE}$ & $\mathrm{B}$ & $\mathrm{SB}$ & $\mathrm{S}$ \\
$\mathrm{NS}$ & $\mathrm{VB}$ & $\mathrm{MB}$ & $\mathrm{SB}$ \\
\hline
\end{tabular}

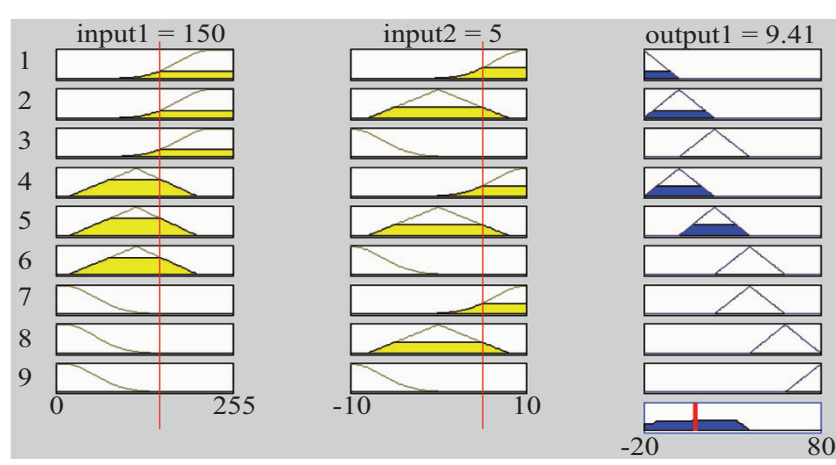

Fig. 12. The inferred output of the DISO system.

output solved by the center-of-gravity method can also be checked by hand calculation as follows:

$$
y=\frac{30 * 5.7461+20 * 15.616+44.223 * 1.637}{5.7461+15.616+1.637}=24.22
$$

\section{DISO}

We assume that the inputs to the system are the environmental light $x_{1}$ and the changing rate of the environmental light $x_{2}$. Where the changing rate of the environmental light ranges between -10 and +10 and is divided into Negative-Small, Zero, and Positive-Small. We further assume that the environmental light can be Small, Medium, and Big. To make the inferred output value homogeneously distribute on all regions (especially, for dark region), the output DN ranges between -20 and 80 and is divided into VS, S, SB, MB, B, and VB. Figure 11 shows the fuzzy sets describing the above. Labels for the membership functions are given in Table 3. The rules base, with its 9 rules, is shown in Table 4. Figure 12 shows the results of the simulation of the rule base by fuzzy inference development environment (MATLAB Fuzzy Logic Toolbox) 


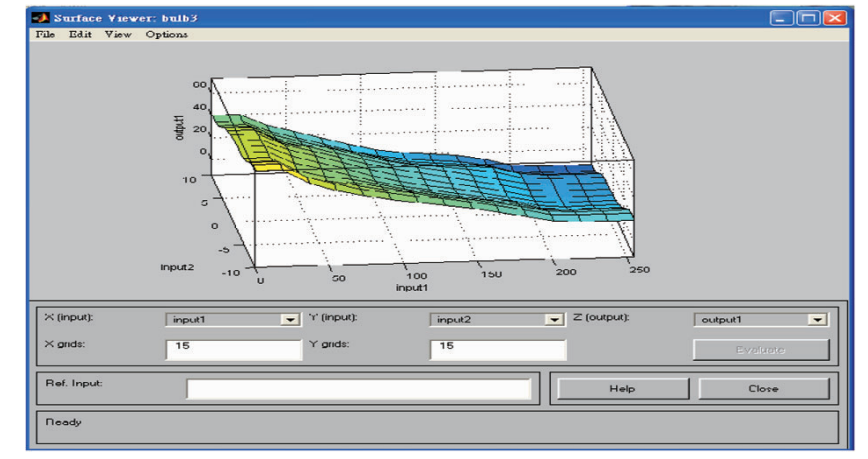

Fig. 13. The result of the simulation of the DISO system using surface viewer of MATLAB fuzzy logic toolbox.

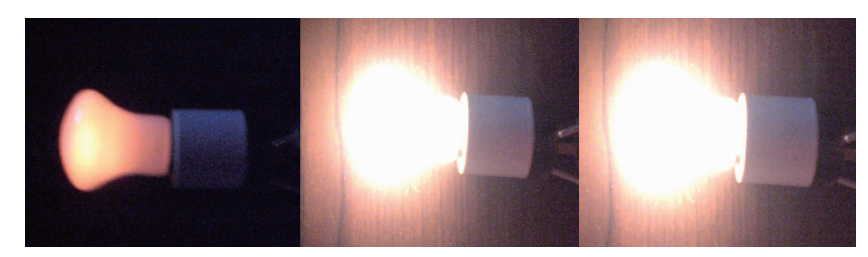

Fig. 14. Bulb responses for control value $\mathrm{DN}=\mathbf{5 , 1 5 , 2 5 .}$

software for the input values of the environmental light $x_{1}=$ 150 and the changing rate of the environmental light $x_{2}=5$. The output is about 9.41. Here only six rules are needed to calculate the output. The surface shown in Fig. 13 is the control surface. It means that for every possible value of the two inputs, there is a corresponding output based on the rules. For example, if the environmental light $x_{1}$ and the changing rate of the environmental light $x_{2}$ are given, the control output DN of the system can be obtained immediately.

\section{RESULTS AND DISCUSSION}

The light control system is constructed in a PC base using the 8255 expanding I/O circuit instead of a DAQ card. The $8255 \mathrm{I} / \mathrm{O}$ expanding circuit, sensing circuit, and the output control circuit become the requisite components in the design of a light control system. The resolution of A/D device is 8-bit and the digital outputs used only 6-bit. The tasks of acquiring the input signal, process of the input data, and output of the DN are commanded by using LabVIEW, which is a graphical programming language to accommodate the light control system.

Figure 14 shows the corresponding bulb responses for the control value $\mathrm{DN}=5,15,25$ respectively. Figure 15 shows the change of the lightness of the bulb against the variations of the environment lightness.

The front panel and the block diagram of the LabVIEW program are shown in Fig. 16. At begin, we create a combo box with two items (SISO and DISO) in the front panel of LabVIEW, shown in the upper part of Fig. 16. To simplify the whole program, we make two sub-vi's icons denoted as FLC

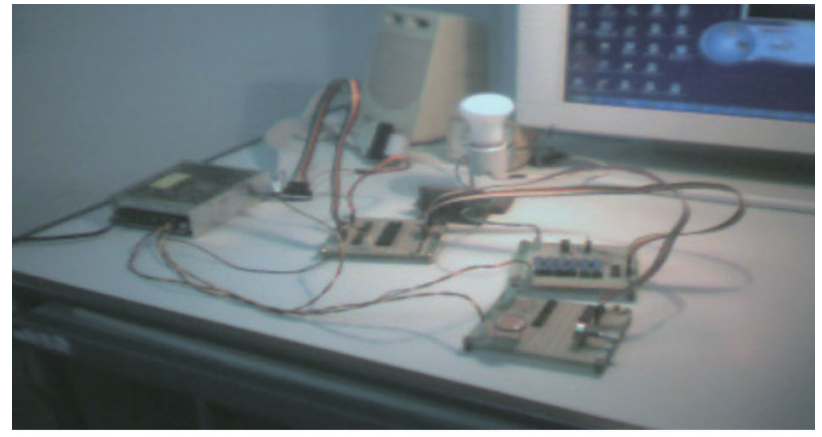

Bright

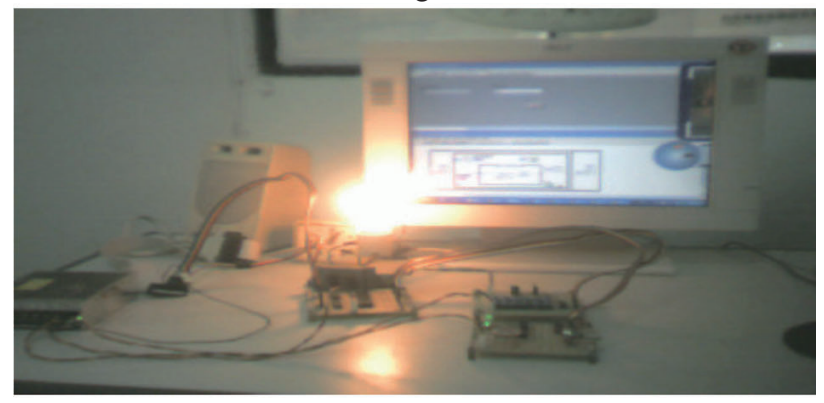

Moderate

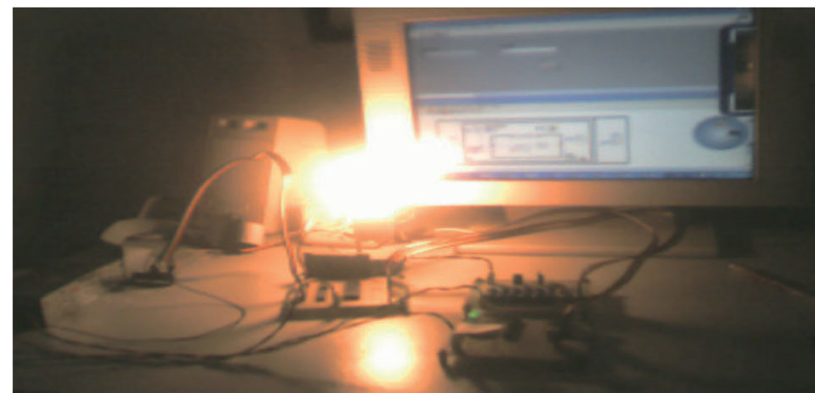

Dark

Fig. 15. Bulb output response against different environmental light.
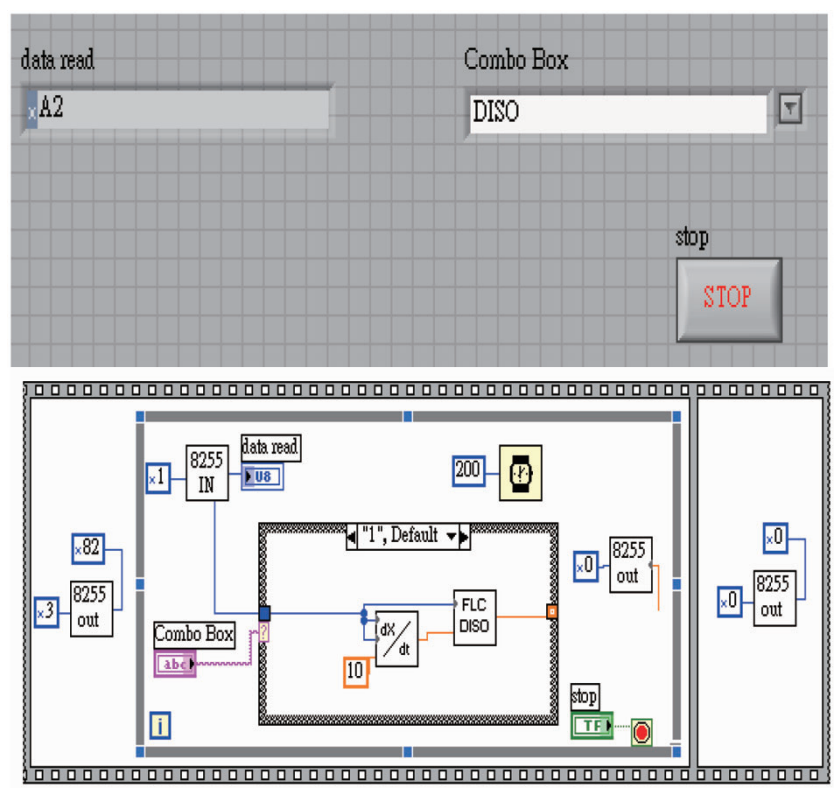

Fig. 16. Front panel and block diagram in LabVIEW. 
SISO and FLC DISO to do the corresponding job of the FLC, shown in the lower part of Fig. 16. After that, we can easily run the LabVIEW program and choose the item in the combo box and even switch the item to another one during the execution of the LabVIEW program.

The main features in the design of the light control system using the LabVIEW are:

(1) Low cost.

(2) Easy implemented in a PC without any DAQ card.

(3) Incorporate MATLAB Fuzzy Logic Toolbox with LabVIEW program.

Javidbakht [1] used fuzzy controller with the SISO sytem to dim the fluorescent lamp based on the availability of the outside light. And Zhang [7] adopted fuzzy controller with the DISO system to distinguish environmental interferences, avoiding the fault action and jittery and improving the reliability. In this paper, both the SISO system and the DISO system are discussed respectively in the fuzzy controller. The experimental results show, due to the light signal and the changing rate are considered, the DISO system responses faster than the SISO system when environmental light changes suddenly. It has also verified the effectiveness and robustness of the proposed fuzzy controller.

\section{CONCLUSIONS}

Important hardware such as the light sensing circuit and the dimmer with relays (like parallel-resistor circuit) were designed and proved work well. The control device can be used to adjust the light level of the bulb, making relays engaged or disengaged and saving the power. The device is reliable and convenient for maintenance.

The control of the bulb light system using a fuzzy logic controller is presented. Both the SISO system and the DISO system are discussed in the fuzzy logic controller and the LabVIEW program incorporated with MATLAB Fuzzy Logic Toolbox for the FLC is carried out. The experimental results have shown the satisfactory response of the bulb system against sudden change of the environmental light. This intelligent dimming device can also be applied to dim the bulb of the streetlights or car's headlights automatically according to environmental light, which worked reliably and had obtained good effect of energy saving.

\section{REFERENCES}

1. Javidbakht, Saeid, Design of a Controller to Control Light Level in a Commercial Office, M.S.Egr. Thesis, Department of Electrical Engineering, Wright State University, pp. 1-106 (2007).

2. Mamdani, E. M., "Application of fuzzy algorithms for control of simple dynamic plant," IEE Proceedings, Vol. 121, No. 12, pp. 1585-1588 (1974).

3. Mamlook, R., Tao, C. W., and Thompson, W. E., "An advanced fuzzy controller," Fuzzy Sets and Systems, Vol. 103, pp. 541-545 (1999).

4. Takagi, T. and Sugeno, M., "Fuzzy identification of systems and its applications to modeling and control," IEEE Transactions on Systems, Man and Sybernetics, Vol. 15, No. 1, pp. 116-132 (1985).

5. Van der Wal, A. J., "Application of fuzzy logic control in industry," Fuzzy Sets and Systems, Vol. 74, pp. 33-41 (1995).

6. Wu, Z. Q., Wang, P. Z., and Wang, H. H., “A rule self-regulating fuzzy controller," Fuzzy Sets and Systems, Vol. 47, pp. 13-21 (1992).

7. Zhang, C., Cui, N., Zhong, M., and Cheng, Z., "Application of Fuzzy Decision in Lighting Control of Cities," 44th IEEE Decision and Control Conference, Seville, Spain, pp. 4100-4104 (2005). 